\title{
ORIGINAL
}

\section{LA FLUORACIÓN DE AGUAS EN LA REGIÓN DE MURCIA}

\author{
María Dolores Marín Camaches (1), Francisco Pacheco Martínez (1), Remedios Martínez Gambín (1), \\ Elisa Gómez Campoy (2) y Carolina Gutierrez Molina (2)
}

(1) Laboratorio de la Consejería de Sanidad y Política Social.

(2) Sección de Sanidad Ambiental de la Consejería de Sanidad y Política Social.

\section{RESUMEN}

Fundamento: El objeto de este trabajo es exponer las concentraciones naturales del ion flúor en las aguas de abastecimiento público de todos los municipios de la Región de Murcia en el año 1991, así como la evolución de dichas concentraciones en el período 1992-96 en el que se ha producido una fluoración en el suministro de agua como medida sanitaria pública.

Métodos: Los métodos utilizados para las determinaciones han sido el de la valoración potenciométrica mediante adición estándar y el de la determinación directa por potenciometría con electrodo selectivo.

Resultados: Los resultados obtenidos indican que se han producido importantes oscilaciones de las cantidades de flúor en las aguas durante los primeros años de fluoración del suministro, sin que sea posible establecer patrones geográficos amplios, para tender hacia una estabilización en los últimos años del período, en los que únicamente en los municipios abastecidos por la depuradora de Letur (que suministra a la zona central y noroccidental de la Región) se han mantenido niveles próximos a $0,8 \mathrm{mg} / \mathrm{L}$, que son los que establece la normativa que acuerda realizar la fluoración.

Conclusiones: De los resultados obtenidos se puede concluir que sólo una parte de los municipios de la Región de Murcia, ha alcanzado el valor óptimo de concentración de flúor en el agua de bebida propuesto por la Comunidad Autónoma $(0,8 \mathrm{mg} / \mathrm{l})$, o valores muy próximos a éste $(0,6-$ $0,7 \mathrm{mg} / \mathrm{l}$ ), durante el período analizado.

Palabras clave: Flúor. Aguas de consumo público. Electrodos selectivos. Salud.

\section{ABSTRACT}

\section{The Addition of Fluoride to Waters in the Region of Murcia}

Background: The purpose of this study is that of expounding upon the natural fluoride ion concentrations in the public water supplies of all of the municipalities in the Region of Murcia in 1991, in addition to the trend in said concentrations throughout the 1992-1996 period, this being a time during which fluoride was added to the water supply as a public health measure.

Methods: The methods employed for the findings were those of potentiometer-based gauging by means of standard addition and direct findings using a potentiometer equipped with an ion-selective electrode.

Results: The findings indicate that major fluctuations have taken place in the amounts of fluoride in these waters throughout the initial years during which fluoride was added to the water supply, without it being possible to establish broad-scoped geographical patterns, to later tend toward stabilization during the final years of the time period in question, in which solely the municipalities supplied by the Letur treatment plant (which supplies the central and northwestern areas of the Region) showed levels nearing 0.8 $\mathrm{mg} / \mathrm{l}$. been maintained, which are those stipulated under the regulations resolving in favor of the addition of fluoride.

Conclusions: We can conclude that only a portion of the municipalities of the Murcia Region has reached the optimun value for the concentration of fluoride in drinking water proposed by the Autonomous Community $(0,8 \mathrm{mg} / \mathrm{liter})$, or values close to it $(0,6-0,7 \mathrm{mg} / \mathrm{liter})$ during the period studied.

Key words: Fluoride. Water drinking. Selective electrodes.

\section{INTRODUCCIÓN}

Correspondencia:

María Dolores Marín Camaches.

Laboratorio de la Consejería de Sanidad y Política Social.

Ronda de I evante, 11.

30008 Murcia.

Fax: (968) 201614.
El flúor que ingerimos procede de la dieta, estando presente fundamentalmente en las aguas de bebida y también en los alimentos en pequeñas cantidades, exceptuando al- 
gunos casos como el té $(50-150 \mathrm{mg} / \mathrm{Kg})$ y pescados (4-5 mg/Kg), o los que proceden de zonas de cultivos ricos en flúor, así como las aguas minerales o vinos de zonas volcánicas, que pueden presentar todos ellos contenidos importantes $^{1.4}$.

El flúor puede catalogarse como un elemento "posiblemente esencial" desde el punto de vista de la nutrición humana, ya que es un oligoelemento imprescindible para la formación de huesos y dientes. Otros aspectos destacables son que hace más resistente el organismo a la descalcificación, interfiere la actividad metabólica de las bacterias de la placa dental y, según la OMS, es el único agente eficaz para la prevención de la caries suministrado a través del abastecimiento de agua ${ }^{5}$.

Para la consecución de los efectos descritos hay que tener en cuenta que su asimilación por el organismo puede estar condicionada por el consumo coincidente de otros elementos con los que pudiera reaccionar, como es el caso del calcio.

A pesar de lo anterior, el flúor sigue siendo un elemento claramente tóxico, en el que sólo la cantidad de las dosis consumidas diferencia los efectos beneficiosos de los perniciosos. Es por ello que resulta necesaria la determinación de las cantidades diarias ingeridas para comprobar si se ajustan a las consideradas como óptimas para el consumo humano, teniendo en cuenta que la dosis óptima es la que se encuentra comprendida entre 0,05 y $0,07 \mathrm{mg} / \mathrm{Kg}$, si se encuentra entre 2 y $4 \mathrm{mg} / \mathrm{Kg}$ es tóxica, y entre 6 y $83 \mathrm{mg} / \mathrm{Kg}$ es letal.

El consumo de $1.200-1.500 \mathrm{ml}$ de agua al día, con un contenido de fluor de $1 \mathrm{mg} / \mathrm{l}$, supone la ingestión de 1,2-1,5 mg/día. Si consideramos que el agua de bebida aporta aproximadamente el 95\% del flúor que consumimos y que distintos trabajos realizados sobre este tema coinciden en afirmar que la fluoración del agua en concentraciones de entre 1 y $2 \mathrm{mg} / \mathrm{l}$ consigue una reducción de hasta el $50 \%$ en la incidencia de caries en dentición permanente, podemos comprender el interés que la OMS ha demostrado para la generalización de la fluoración de las aguas $^{49-12}$. La cuantificación económica de los resultados de esta medida demuestra que los beneficios producidos superan claramente los costes que implica, extendiendo además su efecto positivo a todos los habitantes de una región con independencia de su nivel socio-economico ${ }^{13}$.

Debemos pues aceptar que, aunque existen sectores que critican la conveniencia de la fluoración, tanto por una discutida falta de eficacia como por su caracter coercitivo, existen razones fundadas para pensar que actúa como un factor determinante en la prevención de la caries dental, sin olvidar que en el terreno de la prevención siempre hay que considerar múltiples factores y el flúor es tan sólo uno de esos factores.

La OMS establece una serie de requisitos básicos que deben cumplirse para que la fluoración pueda ser considerada una medida eficaz: grado suficiente de desarrollo económico, constancia de que la población bebe agua de la red pública, moderada frecuencia de caries dental en la comunidad, etc.

Entendiendo cumplidos estos requisitos y siguiendo el criterio de dicho organismo, la Comunidad Autónoma de la Región de Murcia estableció, mediante el Decreto $n^{\circ} 86 / 1990$ de 31 de octubre publicado en el BORM de 14 de noviembre de 1990, la obligación de fluorar las aguas potables de consumo público dentro del ámbito de esta región ${ }^{14}$. En la exposición de motivos de la norma se establece que la concentración óptima de fluoruro, de acuerdo con las temperaturas medias de la zona que se encuentran entre $21^{\circ}$ y $26^{\circ} \mathrm{C}$, será de $0,8 \mathrm{mg} / \mathrm{l}$. En consecuencia, y según el art. 3, se entenderá por fluoración de las aguas potables de consumo público el enriquecimiento del nivel natural del ion fluoruro de dichas aguas, hasta alcanzar un nivel óptimo del citado ion, que en ningún caso podrá superar la cifra de un miligramo por litro, medido en muestras de 
agua potable tomadas en el grifo del consumidor.

Este trabajo pretende, en línea con los estudios realizados en otras zonas de España, la determinación de las cantidades de flúor en el agua de abastecimiento de todos los municipios de la Región de Murcia, pero extendiéndose a lo largo de un período que además coincide con los primeros años de la implantación de la fluoración obligatoria como medida sanitaria pública ${ }^{15-18}$.

\section{MATERIAL Y METODOS}

\section{Muestras}

La toma de muestras, todas procedentes del grifo del consumidor, se ha llevado a cabo a lo largo de la red de abastecimiento de agua potable de los distintos municipios de la Región de Murcia. Las muestras se han conservado en frascos de polietileno y las determinaciones se han llevado a cabo en un período de tiempo inferior a 7 días posteriores a la recogida de las muestras, obteniéndose las curvas de calibración correspondientes.

El número de muestras por año no es el mismo en todos los municipios, y su recogida no se ha efectuado en las mismas fechas. Esta circunstancia puede llegar a producir un sesgo en los resultados que haga menos fiables aquellos valores medios anuales que sean el resultado de una muy pequeña cantidad de muestras. En la tabla de resultados se indican con un asterisco los valores medios anuales obtenidos a partir de sólo una muestra.

\section{Instrumentación y Material}

Potenciómetro: PHM 83 AUTOCAL PH METER, Radiometer Copenhagen 901- 417 Recorder Module, Electrodo selectivo de $\mathrm{F}^{-}$ $\mathrm{PH} / \mathrm{mv}$ (Ingold), Electrodo de referencia: vidrio ref. 100-C, CLK 3M refill 9823 Ingold, Agitador magnético (Placa variomag), Reci- pientes de polietileno para almacenar las disoluciones y efectuar las medidas, Imanes, Material usual de laboratorio.

Reactivos. Soluc. madre de Fluoruro NaF P.A. Solución patrón de fluoruro (mg/L). Solución tampón fluoruro (TISAB): $500 \mathrm{ml} \mathrm{H}_{2} \mathrm{O}$ destilada, $58 \mathrm{~g}$ de CINa P.A. $57 \mathrm{ml}$ acido acético glacial P.A. $5 \mathrm{~g}$ de tritiplex IV P.A. Ajustar PH 5 - 5,5 con $\mathrm{NaOH}$ $8 \mathrm{~N}$ llevar a 1 litro con $\mathrm{H}_{2} \mathrm{O}$ destilada.

\section{Métodos utilizados}

- Valoración potenciométrica con curva de calibración para concentraciones altas del ion fluoruro. A 50 mililitros de muestra se le añaden 5 mililitros de TISAB y se efectúa la lectura del potencial en $\mathrm{mV}$ a una temperatura y agitación constante. A continuación se construyc la recta de calibrado de $\mathrm{F}^{-}$sobre papel gráfico semilogarítmico representando los valores del potencial (E . mv) frente a las distintas concentraciones de fluoruro ( $\mathrm{F}^{-}$en $\mathrm{mg} / \mathrm{L}$ ) en un margen elegido de 0,02 hasta $19 \mathrm{mg} / \mathrm{l}$ y se lee la concentración de fluoruro correspondiente al potencial medido para cada muestra a partir de la recta de calibrado. La sensibilidad de la técnica está en el límite de $0,02 \mathrm{mg} / \mathrm{L}$ de $\mathrm{F}^{-}$, por lo que no se debe utilizar para concentraciones menores ${ }^{19-21}$.

- Valoración potenciométrica mediante técnica de adición estándar para concentraciones pequeñas del ion fluoruro. A $25 \mathrm{ml}$ de muestra se añaden $25 \mathrm{ml}$ de TISAB y se fectúa la lcctura del potencial en $\mathrm{mV}$ a una temperatura y agitación constante. Se añade posteriormente una cantidad de solución estándar conocida, que no debe exceder del $1 \%$ del volumen de la muestra, y que se escogerá de tal forma que permita obtener una concentración final entre 2 y 5 veces más elevada que la de la muestra y efectuamos la segunda lectura de potencial en mv. La concentración de flúor desconocida de la muestra $(C x)$ se calcula a partir de la variación de potencial que resulta $(\Delta \mathrm{E})$. 


$$
\mathrm{Cx}=\Delta \mathrm{c} . \mathrm{A}
$$

donde $\Delta \mathrm{c}=$ Concentración de fluor añadido.

$$
\mathrm{A}=\frac{1}{10^{\Delta \mathrm{E} / \mathrm{S}}-1}
$$

donde $\Delta \mathrm{E}=$ Variac. potencial medio

$\mathrm{S}=$ Pendiente del electrodo

$\mathrm{El}$ factor $\mathrm{A}$ como función de $\Delta \mathrm{E}$ se puede obtener en una tabla de incrementos. Dicha tabla ya ha sido calculada para una pendiente teórica a $25^{\circ} \mathrm{C}$ y para un determinado electrodo selectivo de flúor.

\section{RESULTADOS}

Se han preparado una serie de disoluciones patrón en un rango de concentraciones de $0,19 \mathrm{mg} / \mathrm{L}$ a $19 \mathrm{mg} / \mathrm{L}$ de $F^{-}$y se han representado gráficamente los potenciales medidos ( $E$. mv) frente al log de F, lo que nos proporciona la siguiente recta de calibrado

$$
y=-0,017 x+3,304
$$

con un coeficiente de correlación $\mathrm{r}=0,9998$ (figura 1) (22.23 $^{\text {. }}$

Figura 1

Recta de calibrado del $\mathrm{F}^{-}$

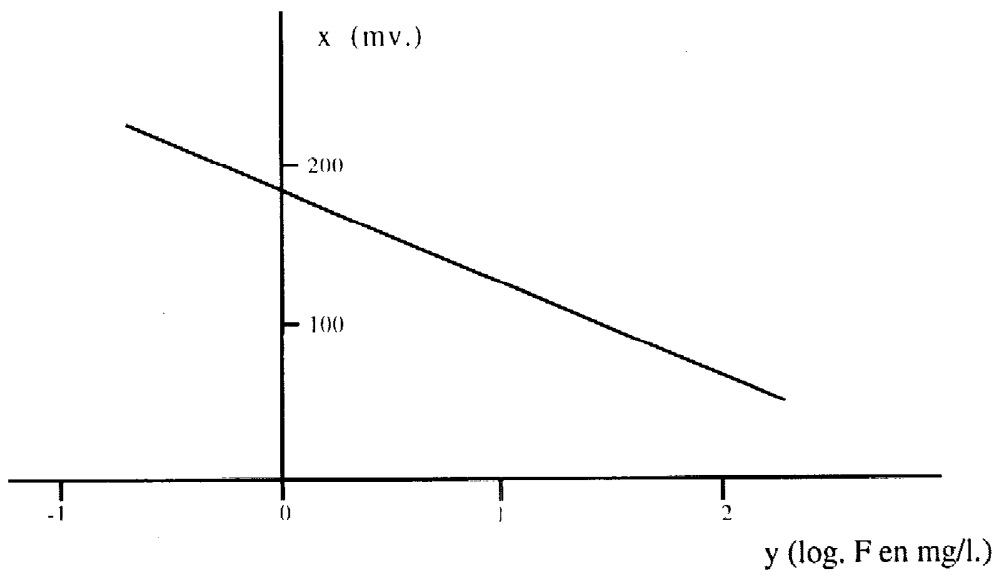

Las variaciones que se han producido en dicha recta a lo largo de los seis años analizados han sido insignificantes, del orden de dos diezmilésimas en los parámetros.

El estudio de precision intraensayo se realizó procesando 10 veces en el mismo día dos disoluciones de concentraciones distintas de fluoruro de $0,3 \mathrm{mg} / \mathrm{l}$ y 0,6 $\mathrm{mg} / \mathrm{l}$ (que son los puntos que definen el intervalo dentro del que se encuentran la mayor parte de los valores de las muestras), obteniendo los resultados reflejados en la tabla 1.
Tabla 1

Imprecisión intraensayo

\begin{tabular}{|ccc|}
\hline$m g / L$ & $\begin{array}{c}\text { Coeficiente } \\
\text { de variación } \\
(\%)\end{array}$ & $\begin{array}{c}\text { Desviación } \\
\text { estándar } \\
m g / l .\end{array}$ \\
\hline 0,3 & 0,22 & 0,00067 \\
0,6 & 0,25 & 0,00152 \\
\hline
\end{tabular}

El estudio de la precisión interensayo se realizó procesando réplicas de una concentración conocida durante 6 días consecutivos. También se hizo con las concentraciones más 
significativas de $0,3 \mathrm{mg} / \mathrm{l}$ y $0,6 \mathrm{mg} / 1$ llegando a los resultados que se reflejan en la tabla 2 .

De dichos datos se concluye que, con un grado de confianza del $95 \%$, los valores reales de fluor en las muestras estarán incluidos en un intervalo de $+/-0,02 \mathrm{mg} / \mathrm{l}$ del valor obtenido en el análisis.

Los resultados del estudio se muestran en la tabla 3.
Tabla 2

Imprecisión interensayo

\begin{tabular}{|ccc|}
\hline$m g / L$ & $\begin{array}{c}\text { Coeficiente } \\
\text { de variación } \\
(\%)\end{array}$ & $\begin{array}{c}\text { Desviación } \\
\text { estándar } \\
m g / L\end{array}$ \\
\hline 0,3 & 0,47 & 0,00140 \\
0,6 & 0,59 & 0,00352 \\
\hline
\end{tabular}

Tabla 3

Contenido medio anual de flúor en aguas de abastecimiento (mg/l)

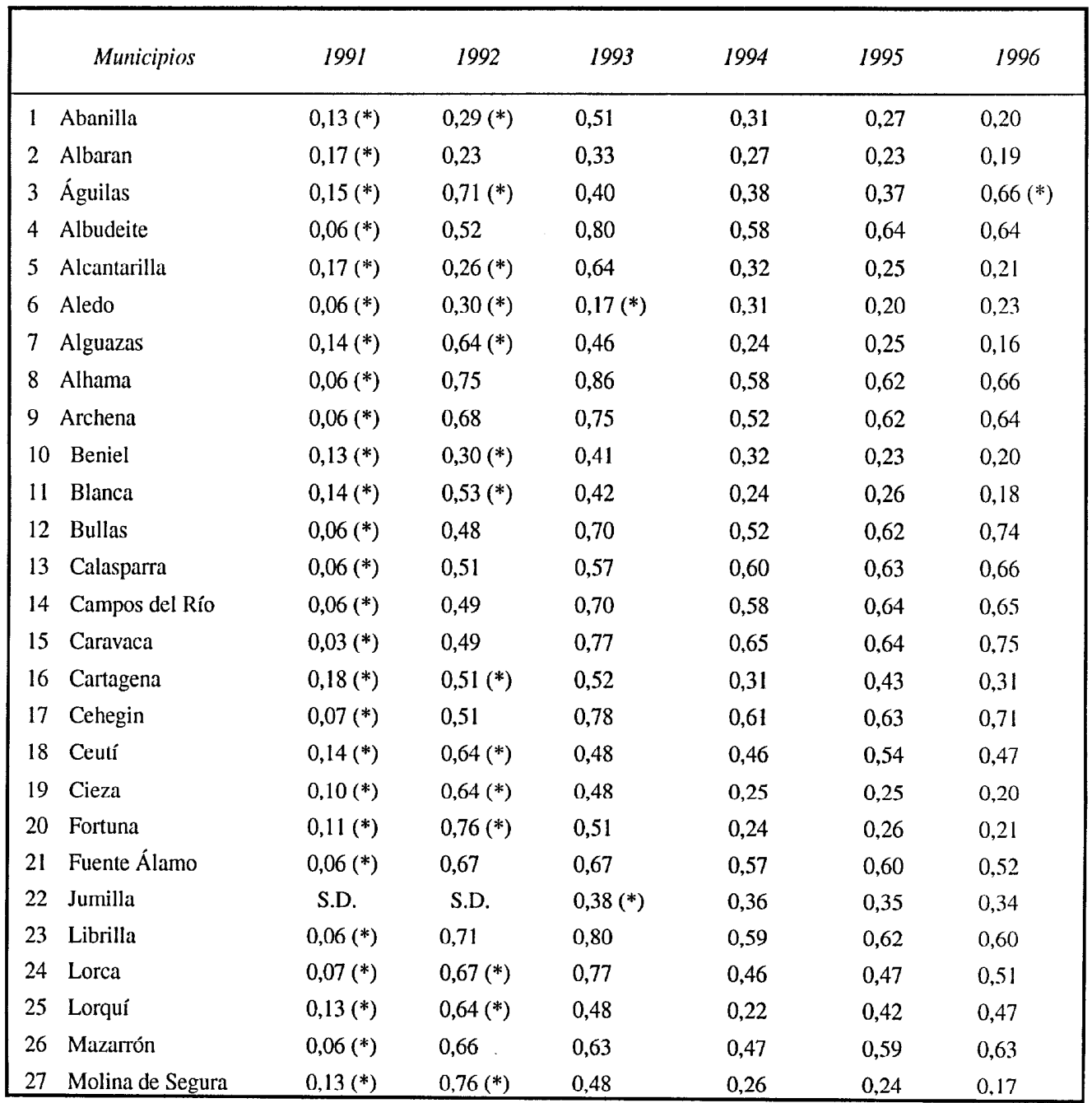


Tabla 3 (Cont.)

\begin{tabular}{|c|c|c|c|c|c|c|c|}
\hline & Municipios & 1991 & 1992 & 1993 & 1994 & 1995 & 1996 \\
\hline 28 & Moratalla & $0,06(*)$ & 0,58 & 0,52 & 0,49 & 0,64 & $0,69\left(^{*}\right)$ \\
\hline 29 & Mula & $0,06\left(^{*}\right)$ & 0,61 & 0,60 & 0,62 & 0,65 & 0,61 \\
\hline 30 & Murcia & $0,13\left(^{*}\right)$ & S.D. & 0,46 & 0,29 & 0,22 & 0,15 \\
\hline 31 & Ojos & $0,06\left(^{*}\right)$ & 0,69 & 0,74 & 0,53 & 0,61 & 0,60 \\
\hline 32 & Pliego & $0,07\left(^{*}\right)$ & 0,60 & 0,67 & 0,59 & 0,67 & 0,64 \\
\hline 33 & Pto. Lumbreras & $0,18\left(^{*}\right)$ & $0,67\left(^{*}\right)$ & 0,44 & 0,33 & 0,42 & 0,29 \\
\hline 34 & Ricote & $0,06\left(^{*}\right)$ & 0,66 & 0,70 & 0,52 & 0,63 & 0,58 \\
\hline 35 & San Javier & $0,19\left(^{*}\right)$ & $0,25\left(^{*}\right)$ & 0,33 & 0,23 & 0,24 & 0,18 \\
\hline 36 & San Pedro del P. & $0,19\left(^{*}\right)$ & $0,26\left(^{*}\right)$ & 0,29 & 0,23 & 0,24 & 0,19 \\
\hline 37 & Santomera & $0,14\left(^{*}\right)$ & $0,18\left(^{*}\right)$ & 0,33 & 0,26 & 0,24 & 0,17 \\
\hline 38 & Torre Pacheco & $0,18\left(^{*}\right)$ & 0,54 & 0,30 & 0,23 & 0,24 & 0,29 \\
\hline 39 & Torres de C., Las & $0,17\left(^{*}\right)$ & $0,70\left(^{*}\right)$ & 0,45 & 0,23 & 0,25 & 0,16 \\
\hline 40 & Totana & $0,06\left(^{*}\right)$ & $0,70\left(^{*}\right)$ & 0,80 & 0,52 & 0,62 & 0,61 \\
\hline 41 & Ulea & $0,06\left(^{*}\right)$ & 0,65 & 0,70 & 0,50 & 0,62 & 0,61 \\
\hline 42 & Unión, La & $0,14\left(^{*}\right)$ & $0,25\left(^{*}\right)$ & 0,55 & 0,31 & 0,35 & 0,29 \\
\hline 43 & Villanueva del S. & $0,06\left(^{*}\right)$ & 0,64 & 0,73 & 0,51 & 0,62 & 0,64 \\
\hline 44 & Yecla & S.D. & S.D. & $0,49\left(^{*}\right)$ & 0.41 & 0.38 & 0,37 \\
\hline 45 & Alcazáres, Los & $0,19\left(^{*}\right)$ & $0,27\left(^{*}\right)$ & 0,36 & 0,27 & 0,25 & 0,21 \\
\hline \multicolumn{2}{|c|}{ Medias anuales } & 0,11 & 0,54 & 0,55 & 0,41 & 0,44 & 0,43 \\
\hline
\end{tabular}

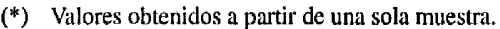
S.D. $=$ Sin datos.

\section{DISCUSIÓN}

Del examen de los resultados se pueden establecer tres períodos claramente diferenciados:

- Durante el año 1991 se obtienen los valores naturales del ion fluoruro, ya que en este momento aún no se fluoraban las aguas.

- Durante los años 1992-93 no se observan pautas geográficas amplias en los valores obtenidos a partir de las muestras, produciéndose grandes oscilaciones en los valores de la mayor parte de los municipios, incluso los geográficamente cercanos, consiguiendo que casi en la mitad de los mismos lleguen a alcanzar en algún momento de este período los valores propuestos de $0,8 \mathrm{mg} / \mathrm{l}$ (ver situación del año 1993 en la figura 2).

- Durante los años 1994-96 se produce una estabilización en los resultados, sin que en ningún caso se alcance durante este período los valores pretendidos por la normativa que acuerda la fluoración. Se observa que los distintos grupos de municipios con valores similares coinciden bastante fielmente con el ámbito gcográfico de las depuradoras que los abastecen (ver situación del año 1995 en la figura 3 ).

Analizando la evolución conjunta de los distintos municipios abastecidos por cada 
Figura 2

Contenido medio anual de flúor en aguas de abastecimiento en mg/L. 1993

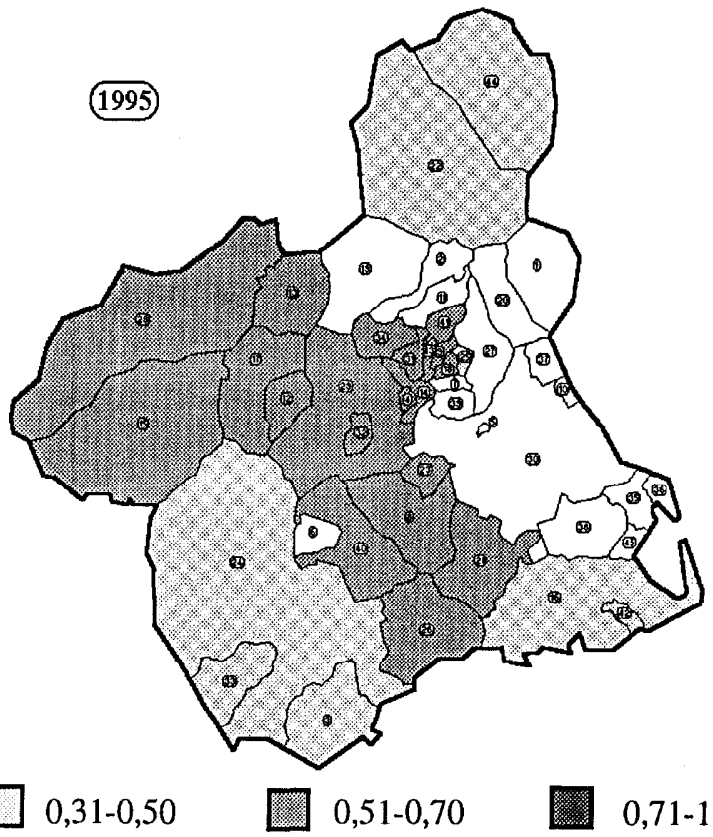

Figura 3

Contenido medio anual de flúor en aguas de abastecimiento en mg/L. 1995

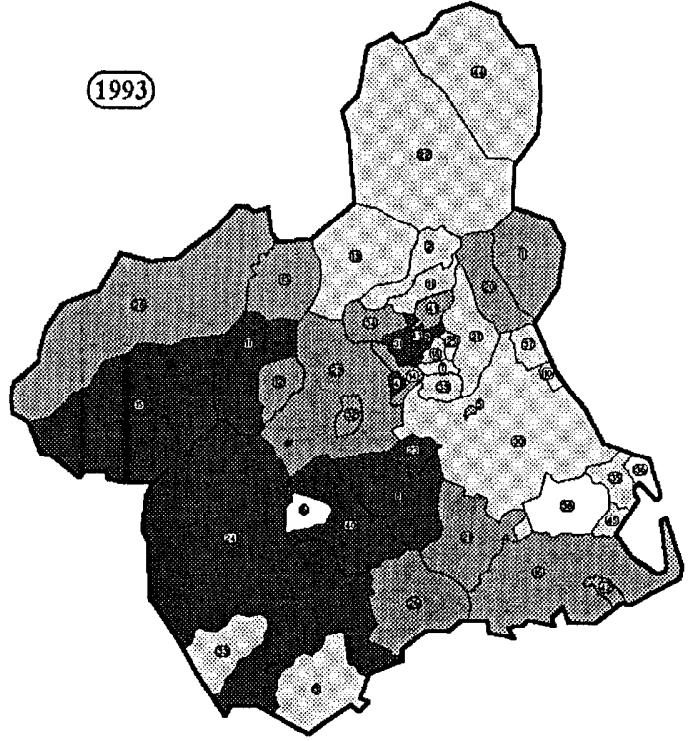
$\square 0-0,30$
$\square 0,31-0,50$
0,51-0,70
0,71-1 
depuradora se pueden establecer tres modelos básicos de comportamiento:

1) Modelo seguido por los municipios abastecidos por la depuradora de LETUR. Los valores suben en los primeros años y se mantienen relativamente elevados a lo largo de todo el período estudiado (entre 0,6 y 0,8 $\mathrm{mg} / \mathrm{l}$ ) (ver figura 4 en la que, para mayor claridad, se han eliminado los municipios más pequeños).

2) Modelo seguido por los municipios abastecidos por la depuradora de la SIERRA DE LA ESPADA. Los valores suben inicialmente hasta $0,5-0,7 \mathrm{mg} / \mathrm{L}$ para descender después a valores próximos a los naturales (excepto los municipios de Lorquí y Ceutí que se mantienen en valores claramente superiores a los naturales al finalizar el período estudiado) (figura 5). A este modelo pueden asimilarse los municipios abastecidos por la depuradora de Lorca, aunque en estos los valores obtenidos al final del período analizado se acercan más a los de los dos municipios antes citados (figura 6).

3) Modelo seguido por los ayuntamientos que se abastecen de las depuradoras de Campotejar y la Pedrera. Los valores suben muy poco inicialmente (entre 0,3 y $0,5 \mathrm{mg} / \mathrm{l}$ ) para bajar a continuación a los valores naturales (figura 7).

4) Por último los municipios abastecidos exclusivamente por Pozos (Yecla, Jumilla y Aledo). Los valores obtenidos permanecen estables en todo el período, oscilando muy ligeramente alrededor de los valores naturales.

De los resultados obtenidos se puede concluir que sólo una parte de los municipios de la Región de Murcia ha alcanzado el valor óptimo de concentración de flúor en el agua de bebida propuesto por la Comunidad Autónoma (0'8 mg/l), o valores muy próximos a este (0'6-0'7 mg/l), durante el periodo analizado.

Figura 4

Evolución del contenido de flúor. Ámbito geográfico de la depuradora de Letur. 1991-96

\section{DEPURADORA DE LETUR}

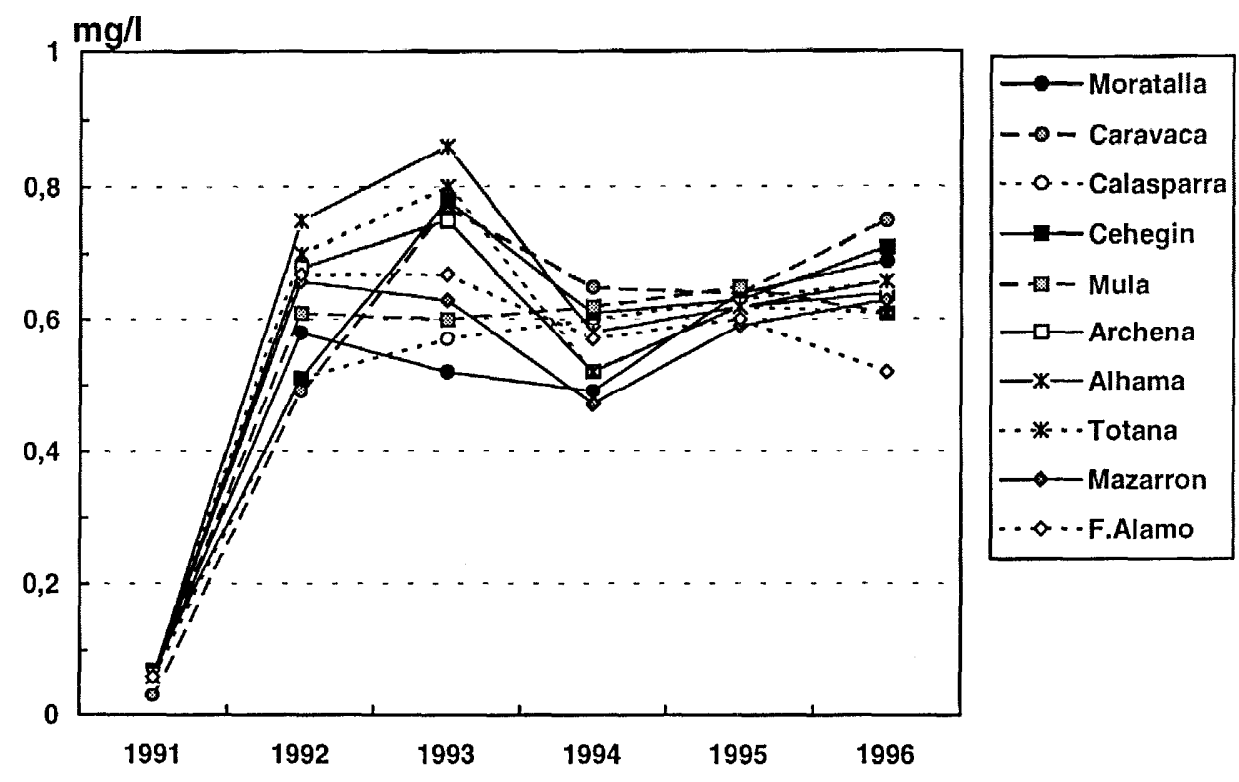


Figura 5

Evolución del contenido de flúor. Ámbito geográfico de la depuradora de Sierra de la Espada. 1991-96

\section{DEPURADORA DE SIERRA DE LA ESPADA}

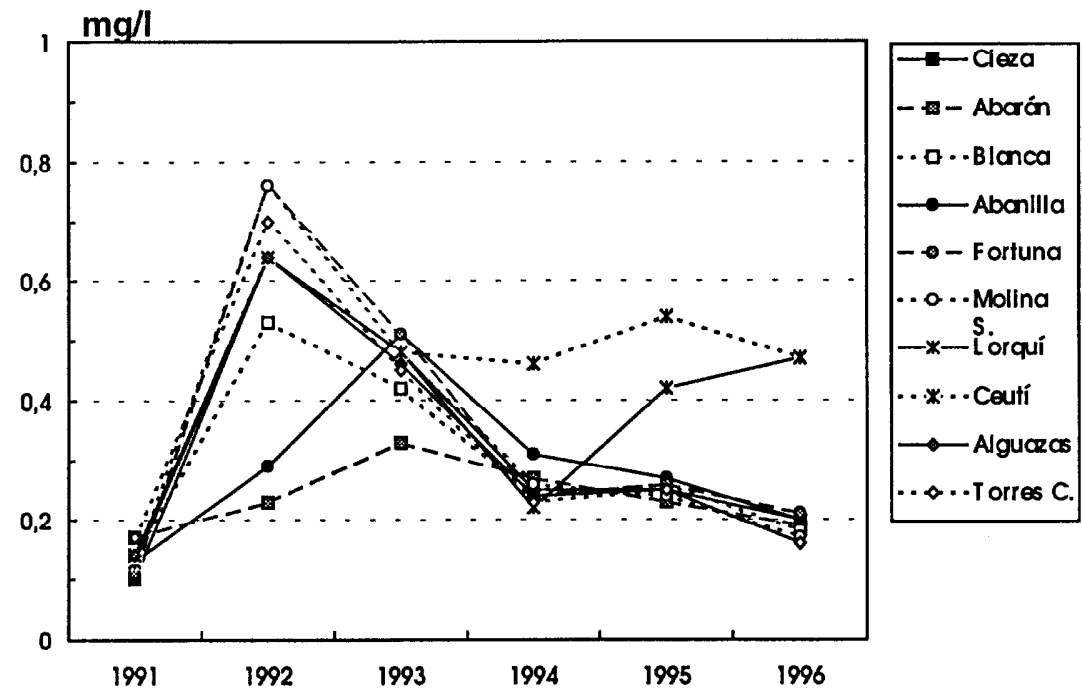

Figura 6

Evolución del contenido de flúor. Ámbito geográfico de la depuradora de Lorca. 1991-96

\section{DEPURADORA DE LORCA}

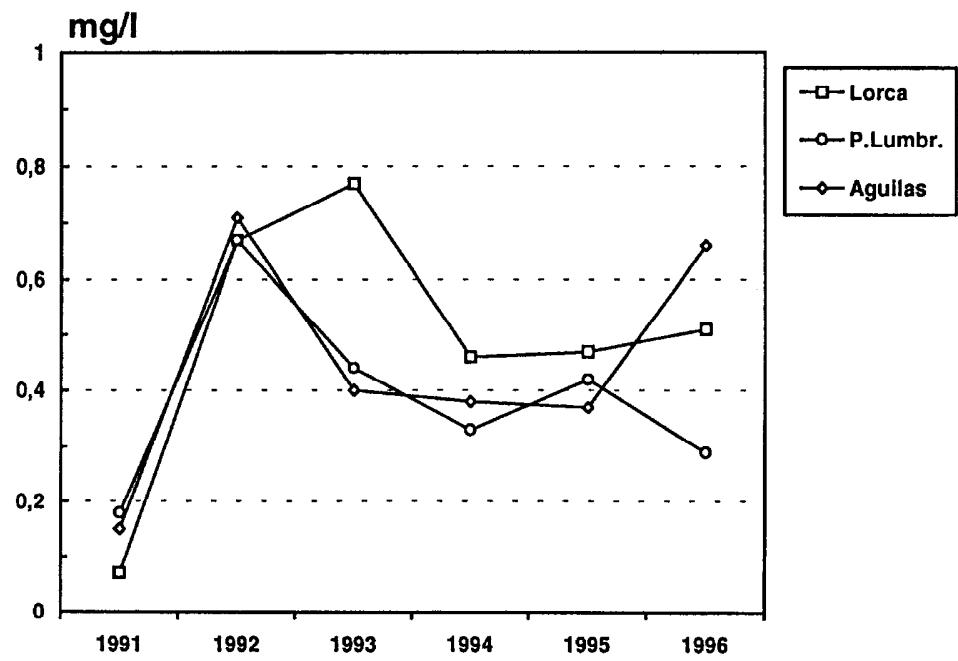




\section{DEPURADORAS DE CAMPOTEJAR Y LA PEDRERA}

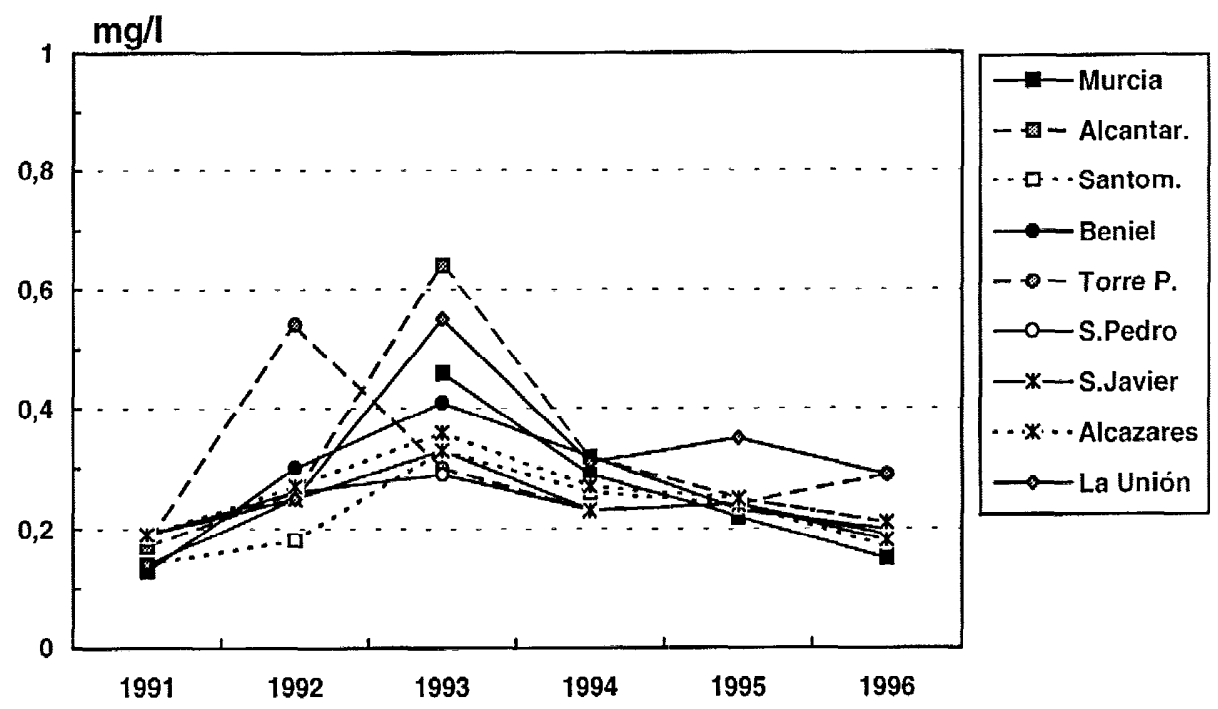

A pesar de que inicialmente no se observan pautas geográficas claras, posteriormente las cantidades de flúor en el agua pasan a depender de forma casi absoluta de cuál sea la depuradora que abastece a cada municipio. El agua obtenida en las redes de los municipios abastecidos por la depuradora de Letur (que suministra agua a la parte central y noroccidental de la Región) es la única que ha mantenido valores situados en el entorno del óptimo propuesto de 0 ' $8 \mathrm{mg} / \mathrm{l}$, mientras que la de aquellos municipios que la reciben de las depuradoras de Sierra de la Espada, Campotejar y La Pedrera ha vuelto a los valores naturales de aproximadamente $0,2 \mathrm{mg} / \mathrm{l}$ después de pasar fugazmente por una fase de leve fluoración, sin que las excepciones lleguen en este caso más allá de los municipios de Ceutí y Lorquí, donde deben haberse producido actuaciones puntuales.

De mantenerse en el futuro la situación descrita, con dos zonas claramente diferenciadas, una con fluoración de aguas y otra sin fluoración, se darían las condiciones adecuadas para efectuar un estudio comparativo de la incidencia de caries dental en cada una de ellas, lo que permitiría comprobar la efectividad de la fluoración llevada a cabo como medida preventiva contra esta enfermedad.

\section{BIBLIOGRAFÍA}

1. Armijo M, Martín J, Armijo F. Contenido en ion flúor de algunas aguas mineromedicinales utilizadas frecuentemente como aguas de mesa. Anal Bromatol 1976; 28: 179-182.

2. Pérez R. Elementos traza en alimentos. Determinación del contenido de fluoruros en tés. Alimentaria 1985 ; 162: 57-61.

3. Comas Font M. Los vinos catalanes, su contenido en flúor y los métodos para su determinación. [tesis doctoral] Universidad Autónoma de Barcelona. Barcelona; 1974.

4. Sánchez A, Robles MP. Flúor y salud. Análisis del contenido de fluoruro en las aguas de con- 
sumo de la provincia de Salamanca. Salamanca: COF; 1989.

5. Schamschula RG, Barmes D.E. Fluoride and Health: dental caries, osteoporosis and cardiovascular disease. Ann Rev Nutr 1981.

6. Augenstein WL, Spoerre D, Kucig K, Hall A, Hall P, Riggs B et al. Fluoride ingestion in children: a review of 87 cases. Pediatrics 1991; 88: 907-912.

7. Real Decreto $1423 / 1982$ de 18 de junio sobre Reglamentación técnico sanitaria para el abastecimiento y control de calidad de aguas potables de consumo público. (BOE núm 154 de 28/6/1982).

8. Muray JJ ed. Appropriate use of fluorides for human health. Ginebra. Organización Mundial de la Salud; 1986.

9. O'Mullane DM. Systemic fluorides. Oral Health Services Research Centre, University Dental School. Cork (Ireland). Adv Dent Res 1994; jul; 8(2): 181-184.

10. Lewis DW, Banting DW. Water fluoridation: current effectiveness and dental fluorosis. Department of Community Dentistry, University of Toronto, Ontario (Canada). Comm Dent Oral Epidemiol 1994; jun; 22(3): 153-158.

11. Nell A, Steinhauser G, Schiestl W, Sperr W. [Measuring drinking water fluoridation in Vorarlbcrg]. Abteilung fur Zahnerhaltung, Universitatsklinic fur Zahn-, Mund- und Kieferheilkunde, Wien. Wien Klin Wochenschr 1993; 105(6): 172-175.

12. Nell A, Sperr W. [Analysis of the fluoride content of drinking water in Austria 1993]. Abteilung fur Zahnerhaltung, Universitatsklinic fur Zahn-, Mund- und Kieferheilkunde, Wien. Wien Klin Wochenschr 1994; 106 (19): 608614.

13. Millán Moleón MT, Gálvez Díaz AJ, Gómez Gracia E, García Rodríguez A, y Fernández-Cre- huet Navajas J. Análisis del Coste-Beneficio de la fluoración de las aguas de abastecimiento de la ciudad de Málaga. Universidad de Granada. Gac Sanit 1991; mar-abr: 82-86.

14. Boletín Oficial de la Región de Murcia. 14 de noviembre de 1990.

15. Arévalo JM, Arranz JF, Barrio RJ, Gómez LA. Valoración de fluoruros en aguas de abastecimiento público en el País Vasco. Anal Bromatol 1984; 36: 275-279.

16. Ezqueta MF, Arcos JM. Contenido de flúor en aguas de abastecimiento de Navarra. Anal Bromato (1975); 27: 333-338.

17 Falcón JT, Hardisson A, Maiquez M. et al. Determinación del contenido de ion fluoruro en las aguas de abastecimiento público de la isla de Tenerife. Anal Bomatol 1984; 36: 265-268.

18. Hidalgo Hidalgo De Cisneros JL, Bueno Garresse E, Pérez Bustamante JA. Determinación del contenido de fluoruro en las aguas potables de la provincia de Cádiz. Anal Bromatol 1982; 34: 13-16.

19. Rodier J. Análisis de las aguas. Barcelona: Omega 1981.

20. American Public Health Association. Standard Methods For The Examination of Water and Wastewater 17 ed. Washington (1989).

21. Korita J, Stulik K. Ion-selective electrodes. 2 ed. Cambridge: Cambridge University Press 1983.

22. San Miguel A, Domínguez E y Rodríguez MJ. Análisis del fluoruro en aguas de abastecimiento público de la provincia de Lugo. Ann Clin 1992.

23. Fuentes De Frutos JE, Matas Rodríguez MJ, San Miguel A. Concentración de fluoruros en leche de vaca. Relación con el contenido de fluoruros en agua de bebida. Ann Clin 1994. 